\section{Asthmatherapie: Was bringt die Zukunft?}

\section{Das Immunsystem bietet zahlreiche Angriffspunkte, an denen die Therapie des allergischen Asthma bronchiale ansetzen kann. Man versucht heute, die komplexen Mechanismen, die bei der Immunpathogenese des Asthma eine Rolle spielen, für die Entwicklung neuer, spezifisch wirkender Medikamente auszunutzen. Monoklonale Antikörper und Antagonisten verschiedener Rezeptoren sollen die allergievermittelnden Substanzen hemmen.}

Um eine allergische Reaktion zu unterdrücken, besteht die Möglichkeit, sowohl die Mediatoren der Früh- als auch der Spätphase zu blockieren. Dabei sind eine Vielzahl von Mediatorsubstanzen beteiligt: Bei der IgE-vermittelten Sofortreaktion sind es die Mediatoren Histamin, Prostaglandine, Leukotriene und der plättchenaktivierende Faktor (PAF).

Bei der asthmatischen Spätreaktion aktivieren TH2-Lymphozyten über die Freisetzung von Zytokinen (IL-4, IL5) die eosinophilen Granulozyten, die dann weitere Mediatoren wie Leukotriene, Thromboxan oder PAF ausschütten. Die Entwicklung neuer Medikamente zielt darauf $a b$, dieses Geschehen entweder mit monoklonalen Antikörpern oder mit Antagonisten der Rezeptoren für die Mediatoren zu beeinflussen.

\section{Spezifischer Angriffspunkt: die Leukotriene}

Leukotrienrezeptor-Antagonisten verhindern die Bindung von bestimmten Leukotrienen sowohl in der allergischen Früh- wie auch in der Spätreaktion. Aus dieser Substanzklasse ist in Deutschland bereits Montelukast unter dem Namen Singulair ${ }^{\circledR}$ auf dem Markt, Zafirlukast (Firma Zeneca) beispielsweise schon in den USA im Handel. Die 5-Lipoxygenase-Hemmer (z.B. Zileuton, Firma Abbott, in Deutschland noch nicht im Handel) greifen dagegen in die Leukotriensynthese ein.

\section{Spezifischer Angriffspunkt: Immunglobulin E}

IgE spielt bei jeder allergischen Reaktion eine Rolle, was das Interesse daran weckt, IgE mit Hilfe monoklonaler Antikörper außer Kraft zu setzen. Derzeit befindet sich ein Medikament mit dem vorläufigen Namen E25 in der klinischen Phase III. Es handelt sich da- bei um einen Antikörper gegen IgE, der von den Firmen Novartis, Genentech und Tanox Biosystems entwickelt wurde. Erste Erfolge erreichte man mit E25 bereits in einer Phase-II-Studie mit 317 Patienten, die an mäßigem bis schwerem allergischen Asthma litten (vgl. Allergo Journal Nr. 5/99, S. 209).

$\mathrm{Zu}$ den weiteren Substanzen, mit denen man derzeit experimentiert, zählen Antagonisten von Zytokinen (IL-4) oder Thromboxan sowie Hemmstoffe der Phosphodiesterasen.

\section{Spezifisches Wirkprinzip wirklich vorteilhaft?}

Der Unterschied dieser Substanzen zu den bisher in der Asthmatherapie breit eingesetzten Steroiden liegt in der Spezifität der Wirkung. Welche Vorteile diese neuen spezifisch wirkenden Substanzen gegenüber den relativ unspezifischen, antientzündlich wirkenden Steroiden haben werden, muß sich noch zeigen. Auch über mögliche Risiken, die in dieser Spezifität stecken, muß nachgedacht werden.

(jn)

\section{Asthmarisiko: Mutter entscheidet}

\author{
Das Risiko, an Asthma zu erkranken, wird zum Teil vererbt. Doch \\ nicht jeder Elternteil überträgt diese Disposition auf seine Kinder \\ im selben Ausmaß - die Mutter scheint dominant zu sein.
}

Wie zwei Untersuchungen mit 3853 vierjährigen Kindern und 2672 Schulkindern zwischen 6 und 16 Jahren belegen, sind die von der Mutter stammenden Faktoren von größerer Bedeutung als die Faktoren, die der Vater mitbringt. Hatten die untersuchten Kinder asthmatische Beschwerden, so ermittelte man, ob die Geschwister oder Halbgeschwister ebenfalls an Asthma litten. Unter den Vollgeschwistern (gleicher Vater und gleiche Mutter) waren dies $37 \%$.

Bei Halbgeschwistern hing das Risiko davon $\mathrm{ab}$, ob sie denselben Vater oder dieselbe Mutter hatten. Bei gleicher Mutter, aber verschiedenen Vä- tern, lag das Asthmarisiko bei $25 \%$. Im umgekehrten Fall, wenn die Halbgeschwister also den gleichen Vater und verschiedene Mütter hatten, betrug das Risiko nur $13 \%$.

Diese Studien bestätigen damit nicht nur den Einfluß genetischer Faktoren auf das Asthmarisiko. Sie zeigen, daß die Mutter größeren Einfluß ausübt als der Vater. Ähnliche Untersuchungen in jüngerer Zeit kamen zu den gleichen Ergebnissen - und zwar nicht nur für das Asthma bronchiale, sondern auch für das Risiko, ein atopisches Ekzem zu entwickeln.

(jn) 